\title{
Native Language Experience Shapes Neural Basis of Addressed and
}

\section{Assembled Phonologies}

Leilei Mei ${ }^{1,2}$, Gui Xue ${ }^{3}$, Zhong-Lin $\mathrm{Lu}^{4}$, Qinghua $\mathrm{He}^{5}$, Miao Wei ${ }^{6}$, Mingxia Zhang ${ }^{3}$, Qi Dong ${ }^{3}$, Chuansheng Chen ${ }^{2}$

${ }^{1}$ Guangdong Key Laboratory ofMental Health and Cognitive Science, Center for Studies of Psychological Application and School of Psychology, South China Normal University, Guangzhou, China

${ }^{2}$ Department of Psychology and Social Behavior, University of California, Irvine, California, USA

${ }^{3}$ State Key Laboratory of Cognitive Neuroscience and Learning \& IDG/McGovern Institute for Brain Research, Beijing Normal University, Beijing, China

${ }^{4}$ Center for Cognitive and Behavioral Brain Imaging and Department of Psychology, Ohio State University, Columbus, Ohio, USA

${ }^{5}$ Faculty of Psychology, Southwest University, Chongqing, China

${ }^{6}$ Department of Psychology, University of Southern California, Los Angeles, California, USA

Correspondence should be sent to:

Leilei Mei: m11830925@126.com

Phone: $86-20-85216922$

Fax: 86-20-85216412

Or 
Chuansheng Chen: cschen@uci.edu

Phone: 1-949-824-4184

Fax: 1-949-824-3002 


\section{Abstract}

Previous studies have suggested differential engagement of addressed and assembled phonologies in reading Chinese and alphabetic languages (e.g., English) and the modulatory role of native language in learning to read a second language. However, it is not clear whether native language experience shapes the neural mechanisms of addressed and assembled phonologies. To address this question, we trained native Chinese and native English speakers to read the same artificial language (based on Korean Hangul) either through addressed (i.e., whole-word mapping) or assembled (i.e., grapheme-to-phoneme mapping) phonology. We found that, for both native Chinese and native English speakers, addressed phonology relied on the regions in the ventral pathway, whereas assembled phonology depended on the regions in the dorsal pathway. More importantly, we found that the neural mechanisms of addressed and assembled phonologies were shaped by native language experience. Specifically, two key regions for addressed phonology (i.e., the left middle temporal gyrus and right inferior temporal gyrus) showed greater activation for addressed phonology in native Chinese speakers, while one key region for assembled phonology (i.e., the left supramarginal gyrus) showed more activation for assembled phonology in native English speakers. These results provide direct neuroimaging evidence for the effect of native language experience on the neural mechanisms of phonological access in a new language and support the assimilation-accommodation hypothesis.

Keywords: Addressed phonology; Assembled phonology; Word reading; 
Cross-cultural differences; fMRI. 


\section{Introduction}

A key component of reading is to transform visual words into their phonologies (i.e., phonological access). The dual-route model of word reading postulates whole-word mapping and grapheme-to-phoneme mapping as two distinct routes (or mechanisms) of phonological access (e.g., Coltheart et al., 2001; Perry et al., 2007; Reynolds et al., 2011). Both routes operate in parallel in word reading, but the degree of their engagement varies across different types of words within a script as well as across different scripts. Specifically, assembled phonology utilizes grapheme-to-phoneme mapping and is mainly used to read unfamiliar regular words (e.g., "bucolic") and pseudowords (e.g., “grem”), whereas addressed phonology relies on direct associations between visual words and their sounds and is mainly used to read familiar words (i.e., "tree") and exception (or irregular) words (e.g., "pint") (Coltheart et al., 2001; Cummine et al., 2013). In contrast to the dual-route model, the triangle model of reading emphasizes interactive parallel processing of orthographic, phonological, and semantic information (Harm and Seidenberg, 2004; Plaut et al., 1996). According to this model, semantics is crucial for addressed phonology but not for assembled phonology.

A large number of neuroimaging studies have suggested that addressed and assembled phonologies may rely on different neural pathways. These studies compared neural activations associated with different reading materials: familiar words vs. pseudowords (e.g., Carreiras et al., 2007; Fiebach et al., 2002; Hagoort et al., 1999; Mechelli et al., 2003; Price et al., 1996; Taylor et al., 2013), irregular words 
vs. regular words (e.g., Fiez et al., 1999; Mechelli et al., 2005; Nosarti et al., 2010; Schurz et al., 2010), Japanese kanji vs. kana (e.g., Ha Duy Thuy et al., 2004; Nakamura et al., 2005; Sakurai et al., 2000), and Chinese characters vs. pinyin (e.g., Chen et al., 2002; Fu et al., 2002). Meta-analyses on those studies have suggested that the neural pathway associated with addressed phonology may include regions such as the anterior fusiform gyrus, middle temporal gyrus, and angular gyrus (Cattinelli et al., 2013; Taylor et al., 2013). The neural pathway associated with assembled phonology may include the posterior fusiform cortex, inferior parietal cortex, and inferior frontal gyrus. Nevertheless, the results from these previous studies could have been confounded by factors such as visual form, phonology, semantics, and task difficulty. To better control for those confounding factors, our previous study adopted an artificial language training paradigm and trained two matched groups to learn the same artificial language words either through addressed phonology or assembled phonology (Mei et al., 2014). Our study revealed a clear dissociation of the neural pathways for addressed and assembled phonologies. Specifically, addressed phonology relied on the right orbital frontal cortex, middle temporal gyrus, and angular gyrus, whereas assembled phonology depended on the left supramarginal gyrus.

Previous studies have also suggested that the degree of engagement of addressed and assembled phonologies varies across different language systems because of their differences in orthographic transparency. Logographic languages such as Chinese (a nontransparent orthography) do not have letter-phoneme mappings, and consequently 
their phonological access relies on addressed phonology (Chen et al., 2009). In contrast, phonological access in alphabetic languages that map graphemes onto phonemes relies more on assembled phonology than that in logographic languages, especially when the alphabetic languages (e.g., Italian) have shallow orthography rather than deep orthography (e.g., English) (Paulesu et al., 2000). The differences in orthographic transparency across different language systems have been found to have important effects on the neural mechanisms for word reading. It has been reported that key regions for assembled phonology in alphabetic languages, such as the left posterior superior temporal gyrus (STG) and adjacent supramarginal gyrus (SMG), are not involved in reading Chinese (Bolger et al., 2005; Tan et al., 2005). Instead, the left middle frontal gyrus (MFG) is heavily involved for addressed phonology in reading Chinese (Liu et al., 2007; Tan et al., 2005; Tan et al., 2001). Further evidence suggests that functional and structural abnormality in the left MFG may be associated with Chinese dyslexia (Siok et al., 2008; Siok et al., 2004; Siok et al., 2009).

More interestingly, existing research has indicated that an individual's native language can shape the cognitive (Andrews, 1989; Wang et al., 2003) and neural mechanisms (Nakada et al., 2001; Tan et al., 2003) involved in the processing of a second language. To account for the effect of native language on second language learning and processing, Perfetti and colleagues (2007) have proposed an intriguing model that consists of two processes, namely, assimilation and accommodation. The assimilation hypothesis assumes that the human brain will read a second language as if it is the native language and use the neural network for the native language to 
support the second language. For example, Tan et al. (2003) found that Chinese speakers who learned English as a second language showed greater activation in the left MFG (usually more involved in processing Chinese relative to English) than English speakers when reading English words. Furthermore, Nelson et al. (2009) found that, in contrast to the left-lateralized activation in the occipitotemporal cortex when English speakers were reading English words, the bilateral occipitotemporal cortex was activated when Chinese speakers were reading English words, which was similar when they were reading Chinese words. The above two findings support the assimilation hypothesis. The accommodation hypothesis assumes that the brain's reading network must adapt to the features of a new writing system to the extent that those features require different reading processes. Consistent with the accommodation hypothesis, there is evidence that when reading Chinese characters (relative to English words), English speakers who are learning Chinese show greater activation in the right occipitotemporal cortex and left MFG, regions heavily involved in processing Chinese for Chinese speakers (Liu et al., 2007; Nelson et al., 2009).

Although accumulating neuroimaging research provides evidence for the assimilation-accommodation hypothesis by comparing native readers with second-language learners, such studies have two important limitations. First, the comparison between the native language and second language could have been confounded by many factors such as language proficiency, age of acquisition, and learning methods, all of which may have significant effects on the reading neural network (e.g., Chee et al., 2001; Hernandez and Li, 2007). Second, word reading in 
natural languages usually involves the automatic processing of visual form, phonology, and semantics. It is difficult to disentangle the contributions of these linguistic factors.

To overcome those limitations and further test the assimilation-accommodation hypothesis, the present study adopted an artificial language training paradigm that was used in our previous study (Mei et al., 2014), and trained native Chinese and native English speakers to read the same artificial language words through either addressed or assembled phonology. By using an artificial language, we could effectively control or manipulate factors such as language proficiency, age of acquisition, and learning methods. Semantics were excluded to avoid the effect of semantics on the neural mechanisms of phonological access in a new language. In this study, we examined 1) the neural mechanisms of addressed and assembled phonologies in native Chinese speakers (the results for native English speakers were reported in Mei et al. (2014)) by comparing brain activation patterns elicited by addressed phonology vs. assembled phonology; 2) the effect of native language experience on neural mechanisms of addressed and assembled phonologies by comparing brain activation of native Chinese speakers vs. native English speakers. Given their differential experience with addressed and assembled phonologies, we expected that native Chinese speakers would show greater activation in regions for addressed phonology, whereas native English speakers would show more activation in regions for assembled phonology.

\section{Methods}


Subjects

Forty-two native Chinese speakers (21 males; $22.05 \pm 1.85$ years old) and 43 native English speakers (20 males; $21.19 \pm 1.97$ years old) with no prior experience of Korean language participated in this study. Native Chinese speakers and native English speakers were matched on nonverbal intelligence (Raven's Advanced Progressive Matrices) (Raven, 1990), verbal working memory [nonword repetition from Comprehensive Test of Phonological Processing (CTOPP)] (Wagner et al., 1999), and visual-auditory associative learning ability [visual-auditory learning from Woodcock Reading Mastery Tests - Revised (WRMT-R)] (Woodcock, 1987). Within each sample, subjects were divided into two groups: one was trained on "addressed phonology" and the other on "assembled phonology". These groups were matched on reading performance in their native languages: Native Chinese speakers' performance was based on Chinese word identification task and word efficiency task; and native English speakers' performance was based on two subtests of WRMT-R (word identification and word attack) and two subtests of the Test of Word Reading Efficiency (TOWRE) (phonemic decoding efficiency and sight word efficiency) (Torgesen et al., 1999) (Table 1).

Native English speakers consisted of 17 monolinguals and 26 bilinguals whose second language was one of alphabetic languages (e.g., Spanish, French, or German) (please see Mei et al., 2014 for details). All native Chinese speakers had been learning English as a second language for about 11 years in school, per Chinese government's educational policies. The two groups of Chinese speakers were matched on English 
reading performance [English word identification (82.14 for addressed group vs. 80.10 for the assembled group, $t(40)=0.55$, n.s.), English word attack (28.95 vs. $30.14, t(40)=0.70$, n.s. $)$, sight word efficiency $(74.29$ vs. $71.81, t(40)=1.19$, n.s. $)$, and phonemic decoding efficiency $(43.48$ vs. $41.95, t(40)=0.65$, n.s. $)]$. Not surprisingly, Chinese speakers' proficiency in English was much lower than that of native English speakers (all $p \mathrm{~s}<.001$ in the four English reading tests).

All subjects had normal or corrected-to-normal vision, had no previous history of neurological or psychiatric disease, and were strongly right-handed as judged by Snyder and Harris's handedness inventory (Snyder and Harris, 1993). Informed written consent was obtained from the subjects before the experiment. This study was approved by the IRBs of the University of California, Irvine, the University of Southern California, and Beijing Normal University.

\section{Materials}

One hundred and twenty artificial language words were used in the study (see Fig. 1 for examples). They were presented in gray-scale and $227 \times 283$ pixels in size. The artificial language words were constructed using 22 Hangul letters (12 consonants and 10 vowels). They consisted of $24 \mathrm{CV}$ and $96 \mathrm{CVC}$ characters. Each consonant appeared 10 times in onset and 8 times in coda, and each vowel was used 10 times. The artificial language words were divided into two groups, one for learning and the other (not trained) for detecting transfer of learning. The two groups of stimuli were strictly matched on number of units (mean number of units $=2.67$ ) and strokes (mean 
number of strokes $=6.15$ ), as well as frequency of each letter.

The sounds of the artificial language words were recorded from a native Korean female speaker. All the sounds were denoised and normalized to the same length (600 ms) and loudness using audacity 1.3 (audacity.sourceforge.net).

\section{Training Procedure and Behavioral Task}

Both native Chinese speakers and native English speakers were asked to learn the association of visual forms and sounds of 60 artificial language words. To contrast the neural bases of addressed and assembled phonologies, we designed two training conditions: addressed-phonology and assemble-phonology training (see Fig. 1A). In the addressed-phonology training condition, subjects were asked to memorize the characters as a whole; whereas in the assembled-phonology training condition, subjects were first taught the pronunciations of the letters and then to assemble the phonology of the characters from the letters. To ensure that subjects focused on the learning of assembled phonology in the assembled-phonology training condition, 30 new characters (untrained characters constructed with letters they already learned) were tested at the end of each day's training. Both types of training lasted for eight days, one hour per day. Except the type of training, all other intervening variables were controlled across the two training conditions and across the two samples (i.e., native Chinese speakers and native English speakers).

At the end of each training day, a word naming task was used to test the acquisition of the association between visual forms and sounds. For the addressed 
group, the word naming task consisted of 60 trained words. For the assembled group, it consisted of 60 trained words and 120 untrained (new) words. Each new word was repeated twice and 30 new words were used on each training day. In the word naming task, each artificial language word was presented for 4 seconds (Days 1-4) or 3 seconds (after Day 4), followed by a 1 second blank. Subjects were asked to read the artificial language words aloud as fast and accurately as possible. The oral responses were recorded. Their accuracy was evaluated by a research assistant by comparing the subjects' responses with the pronunciations used for training.

fMRI Task

After the 8-day training, subjects were scanned while performing a naming task (Fig. 1B). The task consisted of five types of stimuli, namely, trained artificial language words, untrained artificial language words, Chinese words, English words, and alphabetic pseudowords. The Chinese and English materials were included to address other research questions, and excluded from the data analysis in this paper. Each type of material contained 60 items. Stimulus presentation and response collection were programmed using Matlab (Mathworks) and the Psychtoolbox (www.psychtoolbox.org).

The naming task included two runs. Each run consisted of 150 trials, with 30 trials for each condition and with the five types of material pseudo-randomly mixed. A rapid event-related design was used for the naming task. Trial sequences were optimized with OPTSEQ (http://surfer.nmr.mgh.harvard.edu/optseq/) (Dale, 1999). 
For each trial, a word was presented for $1000 \mathrm{~ms}$, followed by a $1000 \mathrm{~ms}$ blank interval. Null events (i.e., fixation) varying from 0.5 to $4 \mathrm{~s}$ (mean $1.2 \mathrm{~s}$ ) were added after each trial to improve design efficiency. All subjects were presented with all five types of materials, and were asked to read each visual word as fast and accurately as possible, and not to respond to the words that they did not know (i.e., subjects in the addressed phonology condition would not read untrained words, and American subjects would not read Chinese words). Subjects' responses were recorded through an MRI-compatible microphone which was connected to a laptop. Each run lasted for $8 \mathrm{~m} \mathrm{10s.}$

Evaluation and Processing of Verbal Responses

Subjects' verbal responses recorded from the scanner were first denoised using audacity 1.3 (audacity.sourceforge.net). The reaction time (RT) for each trial was calculated using the following formula: $\mathrm{RT}=$ response time point $(\mathrm{RTP})-$ trial onset. The RTP was defined as the first time point of 3 continuous points (within the time window of $300-2500 \mathrm{~ms}$ after the stimulus onset) whose intensity was higher than mean +1 standard deviation. The RTP was first automatically identified by an in-house Matlab script, and then manually checked one-by-one by the experimenter.

To calculate the accuracy of the naming task, we had two experimenters evaluate the verbal responses. The agreement rate of the two experimenters was $93.25 \%$ (ranging from $81.67 \%$ to $100 \%$ across subjects) for the artificial language words, suggesting high inter-rater reliability. The items on which the two raters made 
different judgments were discussed and a final decision was made.

\section{MRI Data Acquisition}

Imaging data from the native Chinese speakers were acquired with a $3.0 \mathrm{~T}$ Siemens MRI scanner in the MRI Center at Beijing Normal University, and those from the native English speakers were acquired with a 3.0 T Siemens MRI scanner in the Dana \& David Dornsife Cognitive Neuroscience Imaging Center at University of Southern California. The functional and structural imaging acquisition sequence and parameters were the same for the two MRI scanners. Specifically, a single-shot T2*-weighted gradient-echo EPI sequence was used for functional imaging acquisition with the following parameters: TR/TE $/ \theta=2000 \mathrm{~ms} / 25 \mathrm{~ms} / 90^{\circ}, \mathrm{FOV}=$ $192 \times 192 \mathrm{~mm}$, matrix $=64 \times 64$, and slice thickness $=3 \mathrm{~mm}$. Forty-one contiguous axial slices parallel to the AC-PC line were obtained to cover the whole cerebrum and partial cerebellum. Anatomical MRI was acquired using a T1-weighted, three-dimensional, gradient-echo pulse-sequence. Parameters for this sequence were: $\mathrm{TR} / \mathrm{TE} / \theta=2530 \mathrm{~ms} / 3.09 \mathrm{~ms} / 10^{\circ}, \mathrm{FOV}=256 \times 256 \mathrm{~mm}$, matrix $=256 \times 256$, and slice thickness $=1 \mathrm{~mm}$. Two hundred and eight sagittal slices were acquired to provide a high-resolution structural image of the whole brain.

\section{Image Preprocessing and Statistical Analysis}

Imaging processing was carried out using tools from the FMRIB's software library (www.fmrib.ox.ac.uk/fsl) version 5.0.6. The first 3 volumes of each scan were automatically discarded by the scanner to allow for $\mathrm{T} 1$ equilibrium effects. The 
remaining images were then realigned to compensate for small head movements (Jenkinson and Smith, 2001). Translational movement parameters never exceeded 1 voxel in any direction for any subject or run. The images were denoised using MELODIC independent components analysis within FSL (Tohka et al., 2008). On average 9.53 components were removed from each scanning run. All data were spatially smoothed using a 5-mm full-width-half-maximum Gaussian kernel. The smoothed data were then filtered in the temporal domain using a nonlinear high-pass filter with a 60-s cutoff. A 2-step registration procedure was used whereby EPI images were first registered to the MPRAGE structural image, and then into standard (Montreal Neurological Institute [MNI]) space, using affine transformations with FLIRT (Jenkinson and Smith, 2001) to the avg152 T1 MNI template.

At the first level, the data were fitted with a general linear model within the FILM module of FSL for each subject and each run. Only trials with correct responses were included in the analysis. Incorrect trials were modeled as nuisance variables to avoid the confound of incorrect responses. Trial onsets and durations were convolved with the canonical hemodynamic response function (double-gamma) to generate the regressors used in the general linear model. Temporal derivatives and the 6 motion parameters were included as covariates of no interest to improve statistical sensitivity. Null events were not explicitly modeled, and therefore constituted an implicit baseline. For each subject and each run, two contrast images (trained words minus baseline and untrained words minus baseline) were computed.

A second-level analysis was performed to average across the two runs for each 
subject, using a fixed-effects model. These data were then used for the third-level analyses involving four groups: the addressed group of native Chinese speakers, the assembled group of native Chinese speakers, the addressed group of native English speakers, and the assembled group of native English speakers. In these analyses, we first computed the brain map for each group. Second, we compared the addressed condition with the assembled condition to compute the brain maps of addressed and assembled phonologies, for Chinese and English native speakers separately. Finally, we used a two-way (native language: Chinese and English; training: addressed- and assembled-phonology training) analysis of variance (ANOVA) to examine the effect of native language experience on neural basis of addressed and assembled phonologies. Follow-up simple-effect analysis was used to compute the differences between Chinese and English speakers for each training condition (i.e., addressed and assembled conditions). Group activations were computed using a random-effects model (treating subjects as a random effect) with FLAME stage 1 only (Beckmann et al., 2003; Woolrich, 2008; Woolrich et al., 2004). Unless otherwise indicated, group images were thresholded with a height threshold of $z>2.3$ and a cluster probability of $\mathrm{P}<0.05$, corrected for whole-brain multiple comparisons using the Gaussian random field theory (Worsley, 2001).

\section{Region of Interest Analysis}

Two regions of interest (ROIs), namely the left supramaginal gyrus (SMG) and middle temporal gyrus (MTG), were functionally defined based on the brain map of the native-language-by-training interaction. Each ROI was defined as a region of a 6 
$\mathrm{mm}$ diameter sphere around the local maxima in each cluster. In addition, two regions (i.e., the left and right inferior occipital gyrus) for visual processing were anatomically defined as ROI based on Harvard-Oxford probabilistic atlas (Maximal Probability Threshold: $25 \%$ ) within FSL to estimate the potential differences between the two MRI scanners in signal-to-noise ratio. ROI analyses were performed by extracting parameter estimates (betas) of trained words from the fitted model and averaging across all voxels in the cluster for each subject. Percent signal changes were calculated using the following formula: [contrast image/(mean of run)] $\times$ ppheight $\times$ $100 \%$, where ppheight was the peak height of the hemodynamic response versus the baseline level of activity (Mumford, 2007).

\section{Results}

\section{Behavioral Results}

Behavioral data showed that training significantly improved the naming accuracy of the trained words (higher than $90 \%$ after training) for both native Chinese and native English speakers and for both addressed and assembled conditions (Fig. 2A \& B). Training also significantly improved the accuracy of the untrained words (higher than $85 \%$ after training) in the assembled condition (Fig. 2C). These results (collected outside of the scanner) were confirmed by the behavioral data collected during scanning (Fig. 2D \& E). As expected, subjects in the addressed phonology condition could not correctly name any of the untrained words. These results confirmed our expectation that subjects receiving the two types of training focused on the learning of 
addressed and assembled phonologies, respectively.

We then examined group differences in behavioral performance during learning (Fig. 2). We first examined the data (accuracy) on trained words by performing a three-way repeated measures analysis of variance (ANOVA). It included two between-subject factors (native language: Chinese and English; training: addressedand assembled-phonology training) and one within-subject factor (session: the eight training days). Significant two-way and three-way interactions were found. Specifically, at the early stages of training, regardless of their native language, subjects in the assembled group showed better performance than did those in the addressed group, but the two groups did not differ at the end of training (training-by-session interaction: $F(7,567)=7.92, p<.001$ ). These results were consistent with previous findings that shallow orthography is easier to learn than deep orthography (Aro and Wimmer, 2003; Ellis and Hooper, 2001; Nick et al., 2004). English speakers learned the artificial language better than did Chinese speakers at the early stages of training, but the two types of speakers did not differ at the end of training (native-language-by-session interaction: $F(7,567)=3.44, p=.001$ ). Finally, at the early stages of learning, native English speakers learned assembled phonology better than did native Chinese language, but they did not differ in the learning of addressed phonology (please see Table S1 for the detailed statistics).

For untrained words (the assembled condition), we performed a two-way repeated measures ANOVA (native language and session). Consistent with the results of trained words in the assembled condition, native English speakers performed better 
than native Chinese speakers on untrained words at the early stages of training, but the two types of speakers did not differ at the end of training (native-language-by-session interaction: $F(7,287)=2.28, p<.05$; please also see Table S1 for detailed statistics). These results suggest that English speakers have some superiority in the learning of assembled phonology as compared to Chinese speakers. In other words, native language experience can affect phonological learning in a new language.

We also examined group differences in behavioral performance during scanning. We performed two-way ANOVAs on both reaction time and accuracy of the trained words. Two between-subject factors (native language and training) were included. For reaction time, neither main effects nor interaction were statistically significant (the smallest $p=.114$ ). For accuracy, only the main effect of training was significant. Accuracy was higher for the assembled group than the addressed group regardless of native language $(F(1,81)=23.51, p<.001)$. The main effect of native language $(F(1,81)=1.58, p=.213)$ and the native-language-by-training interaction $(F(1,81)=$ $0.81, p=.371)$ were not significant. These results suggested that RT was matched across the two types of training and across the two types of speakers. In other words, task difficulty was matched across conditions and groups. The differences in accuracy between the two types of training should not affect subsequent fMRI analysis because we only included the correctly named words in the fMRI analysis.

Neural Mechanisms of Addressed and Assembled Phonologies 
Naming trained words elicited activation in the typical reading network for all four conditions (i.e., Chinese speakers' addressed and assembled conditions, English speakers' addressed and assembled conditions), including the anterior cingulate cortex, bilateral prefrontal cortex, occipitoparietal cortex, and occipitotemporal cortex (Fig. S1).

We then examined the neural mechanisms for addressed and assembled phonology by comparing neural activity elicited by trained words in the addressed group with that elicited in the assembled group. As noted in the "Behavioral Results" section, behavioral performance (RT) on trained words was matched across the two groups. The results of native English speakers were reported in Mei et al. (2014). Briefly, the addressed group showed greater activation in the right orbital frontal cortex (OFC) and middle temporal gyrus (MTG) as well as anterior cingulate cortex (ACC), posterior cingulate cortex (PCC), and the right angular gyrus (AG), whereas the assembled group showed greater activation in the left supramarginal gyrus (SMG) [extending to superior parietal lobule (SPL)] (Fig. 3A \& Table 2).

For native Chinese speakers, the addressed group showed greater activation in the ACC, PCC, bilaterial OFC [extending to inferior frontal gyrus (IFG)], MTG, and AG (Fig. 3B \& Table 2), whereas the assembled group showed greater activation in the left precentral gyrus (MNI: $-58,-4,44, Z=3.34$ ) [a relatively liberal threshold $(\mathrm{Z}>$ 2.3, uncorrected) was used]. These results were confirmed by an additional analysis of the contrast of trained words in the addressed group vs. untrained words in the assembled group (to eliminate a potential confound of subjects' use of addressed 
phonology in the trained words in the assembled condition, see Supplementary Results).

Common Activation for Native Chinese and Native English Speakers

To find common activation for native Chinese speakers and native English speakers, we then performed a conjunction analysis, using the procedure suggested by Nichols et al. (2005). Specifically, group maps for the two samples were thresholded individually at $\mathrm{z}=2.3$, binarized, and multiplied, which revealed brain regions that were significantly activated in both samples. For addressed phonology, the two samples showed overlapping activation in ACC, PCC, the right OFC, MTG, and AG (Fig. 3C). For assembled phonology, no regions showed common activation between Chinese and English speakers.

Native Language Experience Shapes Neural Basis of Addressed and Assembled Phonologies

To examine whether native language experience shapes the neural basis of addressed and assembled phonologies, we used a two-way ANOVA (native language and training) to compare neural activities of native Chinese and native English speakers. In this analysis, we focused on the interaction between subjects' native language and training method, which would greatly reduce the scanner effect. This analysis revealed that two regions, the left MTG (a key region for addressed phonology, MNI: -62, -52, -6, Z = 3.20) and SMG (a key region for assembled phonology, MNI: $-38, \quad-44, \quad 42, \quad Z=3.29)$, showed significant 
native-language-by-training interactions (Fig. 4).

We further performed simple effect analysis by comparing neural activity of Chinese speakers with that of English speakers for addressed and assembled phonologies separately. Results showed that for assembled phonology, English speakers showed greater activation than Chinese speakers in the left SMG (extending to SPL) and right IFG (Fig. S2), whereas no regions showed more activation for Chinese speakers. For addressed phonology, Chinese speakers showed more activation than English speakers in the ACC, PCC, left AG, left MTG (extending to ITG), and right ITG, whereas no regions showed greater activation for English speakers. These results suggested that the neural activation in the left SMG and MTG was modulated by native language experience. Furthermore, the less involvement of the left SMG in assembled phonology for Chinese speakers and the less involvement of the left MTG in addressed phonology for English speakers seemed to reflect the assimilation process of the assimilation-accommodation hypothesis (Perfetti and Liu, 2005; Perfetti et al., 2007).

To examine whether the effects of native language experience could have been confounded by potential differences between the two MRI scanners, we extracted percent signal changes in two task-unrelated regions (i.e., the bilateral inferior occipital gyrus). Two-way ANOVAs (native language and training) showed that neither the main effect of native language nor the native-language-by-training interaction was significant (the smallest $p=.188$, Fig. 4), suggesting our results were not confounded by scanner effects. 


\section{Discussion}

Using an artificial language training paradigm with a factorial design, the present study examined the effect of native language experience on the learning of addressed and assembled phonologies in a new language. We found that, for native Chinese speakers, addressed phonology relied more on the ventral pathway (e.g., the OFG and MTG), whereas assembled phonology depended more on the dorsal pathway (e.g., the precentral gyrus). These results are generally consistent with our previous findings for native English speakers (Mei et al., 2014). More importantly, we found that the learning of addressed and assembled phonologies and their neural mechanisms were shaped by native language experience. Behaviorally, compared with native Chinese speakers, native English speakers showed superiority in learning assembled phonology at the early stages of training perhaps because of their long-term experience with assembled phonology. Neuroimaging data showed that native English speakers showed a greater effect in the assembled minus addressed contrast than native Chinese speakers in the left SMG (a key region for assembled phonology), whereas native Chinese speakers showed a greater effect in the addressed minus assembled contrast than native English speakers in the left MTG (a key region for addressed phonology).

To dissociate the neural mechanisms for addressed and assembled phonologies, we relied on the contrast between the trained words in the addressed condition and the 
trained words in the assembled condition as well as the contrast between the trained words in the addressed condition and the untrained words in the assembled condition. Trained words in the addressed group could only be read through addressed phonology, and the untrained words in the assembled group could only be read through assembled phonology. As for trained words in the assembled condition, they could in theory also be read through addressed phonology, but we believe that they were read mainly through assembled phonology for several reasons. First, subjects learned the artificial language words through grapheme-to-phoneme mapping during training and acquired the GPC rules after training, as shown by their ability to name untrained words in the assembled condition. Second, we intermixed the trained words with the untrained words in the naming task during scanning, which would have encouraged the use of assembled phonology. Third, although it has been proposed that the strategy of reading shifts from assembled phonology to addressed phonology as proficiency increases (Binder et al., 2005; Coltheart et al., 2001), the relatively low proficiency (i.e., reaction time $>1300 \mathrm{~ms}$ ) at the end of training in this study suggested the use of assembled phonology. Finally, both sets of analyses (contrasting addressed training with either trained or untrained words in assembled phonology) showed that addressed phonology relied on regions in the ventral pathway (e.g., the OFC and MTG), whereas assembled phonology relied on regions in the dorsal pathway (e.g., the PCG and SMG). These results were consistent with previous studies (Cattinelli et al., 2013; Jobard et al., 2003), and were mostly compatible with both the dual-route model (Coltheart et al., 2001) and the triangle model of reading (Harm and 
Seidenberg, 2004; Plaut et al., 1996; please see Taylor et al., 2013 for a recent meta-analysis).

It should be noted that we found extensive activation in the right hemisphere for addressed phonology relative to assembled phonology. Consistent with our results, several previous studies have also reported activation in the right hemisphere for addressed phonology as shown in the contrasts of Chinese characters minus pinyin (Chen et al., 2002), Kanji minus Kana (Ino et al., 2009; Thuy et al., 2004), and words minus pseudowords (Carreiras et al., 2007; Hagoort et al., 1999; Ischebeck et al., 2004; Mechelli et al., 2003). However, many other studies have only observed activation in the left hemisphere for addressed phonology (Cattinelli et al., 2013; Jobard et al., 2003; Taylor et al., 2013).

There are two possible explanations of the heavy involvement of the right hemisphere in addressed phonology in our study. First, the proficiency in the artificial language was still relatively low even after extensive training. Consequently, regions in the right hemisphere were recruited to process the newly acquired words. Consistent with this view, there is evidence that bilinguals additionally recruit regions in the right hemisphere to process the less proficient language (Chee et al., 2001; Stein et al., 2009), especially when their proficiency is low (Raboyeau et al., 2004). Second, according to the hemispheric specialization view (Hellige et al., 2010), the left and the right hemispheres are specialized for processing, respectively, part versus whole (Rossion et al., 2000) and feature versus holistic information (Grill-Spector, 2001). Therefore, the involvement of the right hemisphere in addressed phonology 
was perhaps due to the fact that the method of addressed-phonology training emphasized holistic processing (whole-word mapping) subserved by the right hemisphere (Mei et al., 2013).

In addition, we found that the left MTG was deactivated during the processing of the artificial language words (with no semantics) relative to rest in this study. One possible explanation is that the left MTG is deactivated for verbal material lacking semantics but activated for that with semantics. This explanation is consistent with previous findings that the left MTG is deactivated for pseudowords but activated for words (Ischebeck et al., 2004), and that it is deactivated during word reading in non-proficient second language learners (whose semantic access is less efficient) but activated during word reading in native speakers (Yokoyama et al., 2009).

Our study provided the first experimental evidence for the effect of native language experience on the neural mechanisms for addressed and assembled phonologies in a new artificial language. Previous studies have suggested that phonological access in Chinese and alphabetic languages (e.g., English) relies differentially on the neural pathways of addressed and assembled phonologies (Bolger et al., 2005; Chen et al., 2009; Tan et al., 2005). Previous studies have also suggested that the neural mechanisms of word reading in a second language can be shaped by the native language (Nakada et al., 2001; Nelson et al., 2009; Tan et al., 2003). Nevertheless, those studies rely on the comparison between native language readers and second language readers (e.g., Liu et al., 2007; Nelson et al., 2009; Tan et al., 2003), which might have been confounded by many factors such as language 
proficiency, age of acquisition, and learning methods. In addition, previous research used natural languages, which may involve automatic co-activation of visual features, form-sound mapping, and semantics. To overcome those limitations, the present study adopted an artificial language training paradigm to control for language proficiency, age of acquisition, and learning methods, and excluded semantics to examine specifically visual form-sound associations. We found that, compared with Chinese speakers, English speakers showed superiority in learning assembled phonology perhaps because of their long-term experience with assembled phonology. In contrast, there was no group difference in learning addressed phonology, which relies on the direct association between whole words and their sounds. This finding of similarity in learning addressed phonology made sense because Chinese and English speakers were matched on visual-auditory learning ability based on the WRMT-R.

More importantly, we found that one key region for addressed phonology (i.e., the left MTG) showed greater activation in the addressed condition for native Chinese speakers, whereas one key region for assembled phonology (i.e., the left SMG) showed greater activation in the assembled condition for native English speakers. These results suggest that the neural mechanisms of addressed and assembled phonologies in a new/artificial language are shaped by native language experience. It should be noted that, although the imaging data of native Chinese and native English speakers were collected from two different MRI scanners (albeit the same model), the neural activity in the bilateral inferior occipital gyrus confirmed that there were no systematic differences between the two MRI scanners. In addition, we used a 
native-language-by-training interaction to examine the native language effect, which would also have minimized any potential scanner effect.

Our results suggest that learning to read a new language includes both assimilation and accommodation processes (Perfetti and Liu, 2005; Perfetti et al., 2007). The assimilation-accommodation hypothesis posits that the human brain can either use the neural network for native language to learn a new language (i.e., assimilation) or recruit additional brain regions to accommodate the special requirement of a new language (i.e., accommodation). The assimilation process has been supported by the greater involvement of the left MFG and right occipitotemporal region in the processing of English for Chinese speakers relative to English speakers (Nelson et al., 2009; Tan et al., 2003); and the accommodation process has been supported by the greater involvement of the same two regions in the processing of Chinese relative to English for English speakers (e.g., Cao et al., 2013; Liu et al., 2007; Nelson et al., 2009), respectively. Those studies might lead to the conclusion that Chinese speakers are more likely to assimilate, and English speakers are more likely to accommodate. Our results disconfirmed that possibility. We found that, for both Chinese and English speakers, both assimilation and accommodation processes were important when learning a new language. Specifically, for Chinese speakers, assimilation was evidenced by more activation in the left MTG for addressed phonology relative to assembled phonology and a lack of activation in the left SMG for assembled phonology relative to addressed phonology, whereas accommodation was shown by more activation in the left precentral gyrus for assembled phonology 
relative to addressed phonology. For English speakers, assimilation was shown by more activation in the left SMG for assembled phonology relative to addressed phonology and a lack of activation in the left MTG for addressed phonology relative to assembled phonology, whereas accommodation was shown by more activation in the right MTG for addressed phonology relative to assembled phonology.

Several studies have reported the critical involvement of the left MFG in the processing of Chinese characters (Tan et al., 2005; Tan et al., 2001), although other studies have failed to confirm this finding (e.g., Chee et al., 2003; Chee et al., 2000; Kuo et al., 2003; Lee et al., 2004; Xue et al., 2005; Xue et al., 2004a; Xue et al., 2004b; Zhang et al., 2004). Tan and colleagues further proposed that the left MFG is responsible for addressed phonology in reading Chinese characters (Tan et al., 2005). Another study from the same authors also revealed that Chinese speakers learning English as a second language recruited the left MFG to read words in the second language (Tan et al., 2003). Inconsistent with that study, the present study did not show any differences in the left MFG for addressed phonology in a new language between native Chinese and native English speakers. We did not observe any activation differences in the left MFG even after we lowered the threshold to $Z=2.3$, uncorrected. The inconsistent results might be caused by several important differences between our study and Tan et al. such as language proficiency and task. First, the artificial language proficiency in this study (eight-hour training) is much lower than English proficiency in Tan et al. (12-year formal instruction on English). The low language proficiency might require more involvement of the left prefrontal cortex for 
both Chinese and English speakers in this study (Chee et al., 2001). Second, Tan et al. used a rhyme judgment task, whereas our study used an overt naming task. There is evidence that, compared with the rhyme judgment task, the overt naming task elicits greater activation in the reading neural network (Vogel et al., 2013). Future studies should include various reading tasks and subjects with different levels of second language proficiency to further examine whether the left MFG is involved in the assimilation process.

The current study has two limitations. First, we did not control for second language experience, which might also have an effect on the neural mechanisms of addressed and assembled phonologies. Specifically, Chinese subjects were college students who all had learned English as a second language for an average of 11 years, as mandated by the Chinese government. Although absolute Chinese monolinguals would have been the ideal subjects for this study, they are rare and would not represent typical Chinese students. The actual extent of the effect of Chinese subjects' experience with English on our findings is hard to gauge, but it is probably safe to assume that any differences we found would be underestimates of the true effects of native language experience because Chinese speakers had experience with both addressed and assembled phonologies, whereas English speakers most likely had experience with only assembled phonology. Future research perhaps can specifically recruit a small group of absolute monolinguals to test this conjecture. Future studies can also include bilinguals with the same native language but different second languages (e.g., logographic vs. alphabetic) to examine the effect of second language 
experience on the neural mechanisms of addressed and assembled phonologies.

Second, the artificial language used in this study was different from natural languages in several important aspects, which might limit the generalization of our findings to natural languages. Unlike natural languages, the artificial language used in this study only had a limited vocabulary, which would eliminate some well-documented effects such as the neighborhood effect and regularity effect, and would impede the acquisition of the inherent structures of words such as the combination of letters (i.e., bigram, trigram). Future studies should include subjects with different native languages but the same second language to confirm our findings in the natural language context. In addition, the artificial language in this study did not include semantics. As discussed in Introduction, compared with the dual-route model (Coltheart et al., 2001), the triangle model highlights the importance of semantics in word reading through addressed phonology (Harm and Seidenberg, 2004; Plaut et al., 1996). Future studies should include semantics to examine its effect on the learning of addressed and assembled phonologies and to test the two models of word reading.

In sum, by using an artificial language training paradigm with a factorial design, our study provides experimental evidence for the effect of native language experience on the neural mechanisms of addressed and assembled phonologies in a new language. 


\section{Acknowledgements}

This work was supported by the National Natural Science Foundation of China (31130025), the 973 Program (2014CB846102), A Foundation for the Authors of

National Excellent Doctoral Dissertations of PR China, FANEDD, (grant number 201410), the National Natural Science Foundation of China (31400847), National Science Foundation (grant numbers BCS 0823624 and BCS 0823495), the National Institute of Health (grant number HD057884-01A2), and the 111 Project (B07008). 


\section{References}

Andrews, S., 1989. Frequency and neighborhood effects on lexical access: Activation or search? Journal of Experimental Psychology: Learning, Memory, and Cognition 15, 802-814.

Aro, M., Wimmer, H., 2003. Learning to read: English in comparison to six more regular orthographies. Applied Psycholinguistics 24, 621-635.

Beckmann, C.F., Jenkinson, M., Smith, S.M., 2003. General multilevel linear modeling for group analysis in FMRI. NeuroImage 20, 1052-1063.

Binder, J.R., Medler, D.A., Desai, R., Conant, L.L., Liebenthal, E., 2005. Some neurophysiological constraints on models of word naming. NeuroImage 27, 677-693.

Bolger, D.J., Perfetti, C.A., Schneider, W., 2005. Cross-cultural effect on the brain revisited: universal structures plus writing system variation. Hum Brain Mapp 25, 92-104.

Cao, F., Vu, M., Lung Chan, D.H., Lawrence, J.M., Harris, L.N., Guan, Q., Xu, Y., Perfetti, C.A., 2013. Writing affects the brain network of reading in Chinese: A functional magnetic resonance imaging study. Human Brain Mapping 34, 1670-1684.

Carreiras, M., Mechelli, A., Estevez, A., Price, C.J., 2007. Brain Activation for Lexical Decision and Reading Aloud: Two Sides of the Same Coin? Journal of Cognitive Neuroscience 19, 433-444.

Cattinelli, I., Borghese, N.A., Gallucci, M., Paulesu, E., 2013. Reading the reading brain: A new meta-analysis of functional imaging data on reading. Journal of Neurolinguistics 26, 214-238.

Chee, M.W.L., Hon, N., Lee, H.L., Soon, C.S., 2001. Relative Language Proficiency Modulates BOLD Signal Change when Bilinguals Perform Semantic Judgments. NeuroImage 13, 1155-1163.

Chee, M.W.L., Soon, C.S., Lee, H.L., 2003. Common and Segregated Neuronal Networks for Different Languages Revealed Using Functional Magnetic Resonance Adaptation. Journal of Cognitive Neuroscience 15, 85-97.

Chee, M.W.L., Weekes, B., Lee, K.M., Soon, C.S., Schreiber, A., Hoon, J.J., Chee, M., 2000. Overlap and Dissociation of Semantic Processing of Chinese Characters, English Words, and Pictures: Evidence from fMRI. NeuroImage 12, 392-403.

Chen, C., Xue, G., Mei, L., Chen, C., Dong, Q., 2009. Cultural neurolinguistics. In: Joan, Y.C. (Ed.), Progress in Brain Research. Elsevier, pp. 159-171.

Chen, Y., Fu, S., Iversen, S.D., Smith, S.M., Matthews, P.M., 2002. Testing for Dual Brain Processing Routes in Reading: A Direct Contrast of Chinese Character and Pinyin Reading Using fMRI. Journal of Cognitive Neuroscience 14, 1088-1098.

Coltheart, M., Rastle, K., Perry, C., Langdon, R., Ziegler, J., 2001. DRC: A dual route cascaded model of visual word recognition and reading aloud. Psychological review 108, 204-256.

Cummine, J., Gould, L., Zhou, C., Hrybouski, S., Siddiqi, Z., Chouinard, B., Borowsky, R., 2013. Manipulating instructions strategically affects reliance on the ventral-lexical reading stream: Converging evidence from neuroimaging and reaction time. Brain and Language 125, 203-214.

Dale, A.M., 1999. Optimal experimental design for event-related fMRI. Human Brain Mapping 8, $109-114$

Ellis, N.C., Hooper, A.M., 2001. Why learning to read is easier in Welsh than in English: Orthographic transparency effects evinced with frequency-matched tests. Applied Psycholinguistics 22, 571-599.

Fiebach, C.J., Friederici, A.D., Miller, K., Cramon, D.Y.v., 2002. fMRI Evidence for Dual Routes to the Mental Lexicon in Visual Word Recognition. Journal of Cognitive Neuroscience 14, 11-23.

Fiez, J.A., Balota, D.A., Raichle, M.E., Petersen, S.E., 1999. Effects of Lexicality, Frequency, and 
Spelling-to-Sound Consistency on the Functional Anatomy of Reading. Neuron 24, 205-218.

Fu, S., Chen, Y., Smith, S., Iversen, S., Matthews, P.M., 2002. Effects of Word Form on Brain Processing of Written Chinese. NeuroImage 17, 1538-1548.

Grill-Spector, K., 2001. Semantic versus perceptual priming in fusiform cortex. Trends Cogn Sci 5, 227-228.

Ha Duy Thuy, D., Matsuo, K., Nakamura, K., Toma, K., Oga, T., Nakai, T., Shibasaki, H., Fukuyama, H., 2004. Implicit and explicit processing of kanji and kana words and non-words studied with fMRI. NeuroImage 23, 878-889.

Hagoort, P., Indefrey, P., Brown, C., Herzog, H., Steinmetz, H., Seitz, R.J., 1999. The Neural Circuitry Involved in the Reading of German Words and Pseudowords: A PET Study. Journal of Cognitive Neuroscience 11, 383-398.

Harm, M.W., Seidenberg, M.S., 2004. Computing the Meanings of Words in Reading: Cooperative Division of Labor Between Visual and Phonological Processes. Psychological review 111, 662-720.

Hernandez, A.E., Li, P., 2007. Age of acquisition: Its neural and computational mechanisms. Psychological Bulletin 133, 638-650.

Ino, T., Nakai, R., Azuma, T., Kimura, T., Fukuyama, H., 2009. Recognition and reading aloud of kana and kanji word: An fMRI study. Brain Research Bulletin 78, 232-239.

Ischebeck, A., Indefrey, P., Usui, N., Nose, I., Hellwig, F., Taira, M., 2004. Reading in a regular orthography: An fMRI study investigating the role of visual familiarity. Journal of Cognitive Neuroscience 16, 727-741.

Jenkinson, M., Smith, S., 2001. A global optimisation method for robust affine registration of brain images. Medical Image Analysis 5, 143-156.

Jobard, G., Crivello, F., Tzourio-Mazoyer, N., 2003. Evaluation of the dual route theory of reading: a metanalysis of 35 neuroimaging studies. NeuroImage 20,693-712.

Kuo, W.J., Yeh, T.C., Lee, C.Y., Wu, Y.T., Chou, C.C., Ho, L.T., Hung, D.L., Tzeng, O.J., Hsieh, J.C., 2003. Frequency effects of Chinese character processing in the brain: an event-related fMRI study. NeuroImage 18, 720-730.

Lee, C.-Y., Tsai, J.-L., Kuo, W.-J., Yeh, T.-C., Wu, Y.-T., Ho, L.-T., Hung, D.L., Tzeng, O.J.L., Hsieh, J.-C., 2004. Neuronal correlates of consistency and frequency effects on Chinese character naming: an event-related fMRI study. NeuroImage 23, 1235-1245.

Liu, Y., Dunlap, S., Fiez, J., Perfetti, C., 2007. Evidence for neural accommodation to a writing system following learning. Hum Brain Mapp 28, 1223-1234.

Mechelli, A., Crinion, J.T., Long, S., Friston, K.J., Ralph, M.A.L., Patterson, K., McClelland, J.L., Price, C.J., 2005. Dissociating Reading Processes on the Basis of Neuronal Interactions. Journal of Cognitive Neuroscience 17, 1753-1765.

Mechelli, A., Gorno-Tempini, M.L., Price, C.J., 2003. Neuroimaging Studies of Word and Pseudoword Reading: Consistencies, Inconsistencies, and Limitations. Journal of Cognitive Neuroscience 15, 260-271.

Mei, L., Xue, G., Lu, Z.-L., He, Q., Zhang, M., Wei, M., Xue, F., Chen, C., Dong, Q., 2014. Artificial Language Training Reveals the Neural Substrates Underlying Addressed and Assembled Phonologies. Plos one 9, e93548.

Mei, L., Xue, G., Lu, Z.-L., He, Q., Zhang, M., Xue, F., Chen, C., Dong, Q., 2013. Orthographic transparency modulates the functional asymmetry in the fusiform cortex: An artificial language training study. Brain and Language 125, 165-172. 
Mumford, J., 2007. A Guide to Calculating Percent Change with Featquery. Unpublished Tech Report available at http://mumford.bol.ucla.edu/perchange_guide.pdf.

Nakada, T., Fujii, Y., Kwee, I.L., 2001. Brain strategies for reading in the second language are determined by the first language. Neurosci Res 40, 351-358.

Nakamura, K., Dehaene, S., Jobert, A., Bihan, D.L., Kouider, S., 2005. Subliminal Convergence of Kanji and Kana Words: Further Evidence for Functional Parcellation of the Posterior Temporal Cortex in Visual Word Perception. Journal of Cognitive Neuroscience 17, 954-968.

Nelson, J.R., Liu, Y., Fiez, J., Perfetti, C.A., 2009. Assimilation and accommodation patterns in ventral occipitotemporal cortex in learning a second writing system. Human Brain Mapping 30, 810-820.

Nichols, T., Brett, M., Andersson, J., Wager, T., Poline, J.-B., 2005. Valid conjunction inference with the minimum statistic. NeuroImage 25, 653-660.

Nick, C.E., Miwa, N., Katerina, S., Lorenc, H., Victor, H.P.V.D., Nicoletta, P., Maria-Louisa, T., Michalis, P., 2004. The Effects of Orthographic Depth on Learning to Read Alphabetic, Syllabic, and Logographic Scripts. Reading Research Quarterly 39, 438-468.

Nosarti, C., Mechelli, A., Green, D.W., Price, C.J., 2010. The Impact of Second Language Learning on Semantic and Nonsemantic First Language Reading. Cereb. Cortex 20, 315-327.

Paulesu, E., McCrory, E., Fazio, F., Menoncello, L., Brunswick, N., Cappa, S.F., Cotelli, M., Cossu, G., Corte, F., Lorusso, M., Pesenti, S., Gallagher, A., Perani, D., Price, C., Frith, C.D., Frith, U., 2000. A cultural effect on brain function. Nat Neurosci 3, 91-96.

Perfetti, C., Liu, Y., 2005. Orthography to Phonology and Meaning: Comparisons Across and within Writing Systems. Reading and Writing 18, 193-210.

Perfetti, C.A., Liu, Y., Fiez, J., Nelson, J., Bolger, D.J., Tan, L.-H., 2007. Reading in two writing systems: Accommodation and assimilation of the brain's reading network. Bilingualism: Language and Cognition 10, 131-146.

Perry, C., Ziegler, J.C., Zorzi, M., 2007. Nested Incremental Modeling in the Development of Computational Theories: The CDP+ Model of Reading Aloud. Psychological review 114, 273-315.

Plaut, D.C., McClelland, J.L., Seidenberg, M.S., Patterson, K., 1996. Understanding normal and impaired word reading: Computational principles in quasi-regular domains. Psychological review 103, 56-115.

Price, C.J., Wise, R.J.S., Frackowiak, R.S.J., 1996. Demonstrating the Implicit Processing of Visually Presented Words and Pseudowords. Cereb. Cortex 6, 62-70.

Raboyeau, G., Marie, N., Balduyck, S., Gros, H., Démonet, J.-F., Cardebat, D., 2004. Lexical learning of the English language: a PET study in healthy French subjects. NeuroImage 22, 1808-1818.

Raven, J.C., 1990. Advanced Progressive Matrices: Sets I, II. Oxford: Oxford University Press.

Reynolds, M., Besner, D., Coltheart, M., 2011. Reading aloud: New evidence for contextual control over the breadth of lexical activation. Memory \& Cognition 39, 1332-1347.

Rossion, B., Dricot, L., Devolder, A., Bodart, J.-M., Crommelinck, M., Gelder, B.d., Zoontjes, R., 2000. Hemispheric Asymmetries for Whole-Based and Part-Based Face Processing in the Human Fusiform Gyrus. Journal of Cognitive Neuroscience 12, 793-802.

Sakurai, Y., Momose, T., Iwata, M., Sudo, Y., Ohtomo, K., Kanazawa, I., 2000. Different cortical activity in reading of Kanji words, Kana words and Kana nonwords. Cognitive Brain Research 9, 111-115.

Schurz, M., Sturm, D., Richlan, F., Kronbichler, M., Ladurner, G., Wimmer, H., 2010. A dual-route perspective on brain activation in response to visual words: Evidence for a length by lexicality 
interaction in the visual word form area (VWFA). NeuroImage 49, 2649-2661.

Siok, W.T., Niu, Z., Jin, Z., Perfetti, C.A., Tan, L.H., 2008. A structural-functional basis for dyslexia in the cortex of Chinese readers. Proc Natl Acad Sci U S A 105, 5561-5566.

Siok, W.T., Perfetti, C.A., Jin, Z., Tan, L.H., 2004. Biological abnormality of impaired reading is constrained by culture. Nature 431, 71-76.

Siok, W.T., Spinks, J.A., Jin, Z., Tan, L.H., 2009. Developmental dyslexia is characterized by the co-existence of visuospatial and phonological disorders in Chinese children. Current Biology 19, R890-R892.

Snyder, P.J., Harris, L.J., 1993. Handedness, sex, and familial sinistrality effects on spatial tasks. Cortex 29, 115-134.

Stein, M., Federspiel, A., Koenig, T., Wirth, M., Lehmann, C., Wiest, R., Strik, W., Brandeis, D., Dierks, T., 2009. Reduced frontal activation with increasing 2nd language proficiency. Neuropsychologia 47, 2712-2720.

Tan, L.H., Laird, A.R., Li, K., Fox, P.T., 2005. Neuroanatomical correlates of phonological processing of Chinese characters and alphabetic words: a meta-analysis. Hum Brain Mapp 25, 83-91.

Tan, L.H., Liu, H.-L., Perfetti, C.A., Spinks, J.A., Fox, P.T., Gao, J.-H., 2001. The Neural System Underlying Chinese Logograph Reading. NeuroImage 13, 836-846.

Tan, L.H., Spinks, J.A., Feng, C.M., Siok, W.T., Perfetti, C.A., Xiong, J., Fox, P.T., Gao, J.H., 2003. Neural systems of second language reading are shaped by native language. Hum Brain Mapp 18, 158-166.

Taylor, J.S.H., Rastle, K., Davis, M.H., 2013. Can cognitive models explain brain activation during word and pseudoword reading? A meta-analysis of 36 neuroimaging studies. Psychological Bulletin 139, 766-791.

Thuy, D.H., Matsuo, K., Nakamura, K., Toma, K., Oga, T., Nakai, T., Shibasaki, H., Fukuyama, H., 2004. Implicit and explicit processing of kanji and kana words and non-words studied with fMRI. NeuroImage 23, 878-889.

Tohka, J., Foerde, K., Aron, A.R., Tom, S.M., Toga, A.W., Poldrack, R.A., 2008. Automatic independent component labeling for artifact removal in fMRI. NeuroImage 39, 1227-1245.

Torgesen, J., Wagner, R., Rashotte, C., 1999. Test of word reading efficiency. Austin, TX: Pro-Ed.

Van Essen, D.C., 2002. Windows on the brain: the emerging role of atlases and databases in neuroscience. Current Opinion in Neurobiology 12, 574-579.

Van Essen, D.C., 2005. A Population-Average, Landmark- and Surface-based (PALS) atlas of human cerebral cortex. NeuroImage 28, 635-662.

Van Essen, D.C., Drury, H.A., Dickson, J., Harwell, J., Hanlon, D., Anderson, C.H., 2001. An Integrated Software Suite for Surface-based Analyses of Cerebral Cortex. Journal of the American Medical Informatics Association 8, 443-459.

Vogel, A.C., Petersen, S.E., Schlaggar, B.L., 2013. Matching is not naming: A direct comparison of lexical manipulations in explicit and implicit reading tasks. Human Brain Mapping 34, 2425-2438.

Wagner, R., Torgesen, J., Rashotte, C., 1999. Comprehensive test of phonological processing: CTOPP. Austin, TX: PRO-ED.

Wang, M., Koda, K., Perfetti, C.A., 2003. Alphabetic and nonalphabetic L1 effects in English word identification: a comparison of Korean and Chinese English L2 learners. Cognition 87, 129-149.

Woodcock, R., 1987. Woodcock Reading Mastery Tests-Revised. Circle Pines, MN: American Guidance Service. 
Woolrich, M.W., 2008. Robust group analysis using outlier inference. NeuroImage 41, 286-301.

Woolrich, M.W., Behrens, T.E.J., Beckmann, C.F., Jenkinson, M., Smith, S.M., 2004. Multilevel linear modelling for FMRI group analysis using Bayesian inference. NeuroImage 21, 1732-1747.

Worsley, K.J., 2001. Stastistical analysis of activation images. In: Jezzard, P., Matthews, P.M., Smith, S.M. (Eds.), Functional MRI: An introduction to Methods. Oxford University Press, Oxford.

Xue, G., Dong, Q., Chen, K., Jin, Z., Chen, C., Zeng, Y., Reiman, E.M., 2005. Cerebral asymmetry in children when reading Chinese characters. Cognitive Brain Research 24, 206-214.

Xue, G., Dong, Q., Jin, Z., Chen, C., 2004a. Mapping of verbal working memory in nonfluent Chinese-English bilinguals with functional MRI. NeuroImage 22, 1-10.

Xue, G., Dong, Q., Jin, Z., Zhang, L., Wang, Y., 2004b. An fMRI study with semantic access in low proficiency second language learners. Neuroreport 15, 791-796.

Yokoyama, S., Kim, J., Uchida, S., Miyamoto, T., Yoshimoto, K., Riera, J., Yusa, N., Kawashima, R., 2009. Left middle temporal deactivation caused by insufficient second language word comprehension by Chinese-Japanese bilinguals. Journal of Neurolinguistics 22, 476-485.

Zhang, J.X., Zhuang, J., Ma, L., Yu, W., Peng, D., Ding, G., Zhang, Z., Weng, X., 2004. Semantic processing of Chinese in left inferior prefrontal cortex studied with reversible words. NeuroImage 23, 975-982. 
Table 1 Mean scores on the reading tests and a nonverbal intelligence test

\begin{tabular}{|c|c|c|c|c|c|c|}
\hline \multirow{2}{*}{ Variables } & \multicolumn{2}{|c|}{ Native Chinese speakers } & \multicolumn{2}{|c|}{ Native English speakers } & \multirow{2}{*}{$\mathrm{F}$} & \multirow{2}{*}{$\mathrm{p}$} \\
\hline & Addressed & Assembled & Addressed & Assembled & & \\
\hline Visual-auditory learning & $\begin{array}{c}123.43 \\
(9.17)\end{array}$ & $\begin{array}{l}123.67 \\
(8.58)\end{array}$ & $\begin{array}{l}122.29 \\
(7.33)\end{array}$ & $\begin{array}{c}123.64 \\
(7.89)\end{array}$ & 0.13 & .941 \\
\hline Raven's Advanced Matrices & $\begin{array}{l}27.95 \\
(4.55)\end{array}$ & $\begin{array}{l}27.67 \\
(3.48)\end{array}$ & $\begin{array}{l}24.95 \\
(3.49)\end{array}$ & $\begin{array}{l}26.23 \\
(4.54)\end{array}$ & 2.46 & .069 \\
\hline Nonword repetition & $\begin{array}{l}12.57 \\
(3.28)\end{array}$ & $\begin{array}{l}13.19 \\
(2.96)\end{array}$ & $\begin{array}{l}13.50 \\
(2.20)\end{array}$ & $\begin{array}{l}13.52 \\
(2.94)\end{array}$ & 0.73 & .540 \\
\hline Chinese word identification & $\begin{array}{l}25.14 \\
(5.76)\end{array}$ & $\begin{array}{l}24.52 \\
(7.40)\end{array}$ & & & 0.91 & .764 \\
\hline Chinese word efficiency & $\begin{array}{c}83.33 \\
(12.88)\end{array}$ & $\begin{array}{c}79.43 \\
(11.43)\end{array}$ & & & 1.08 & .305 \\
\hline English word identification & & & $\begin{array}{l}97.76 \\
(4.39)\end{array}$ & $\begin{array}{l}98.73 \\
(4.03)\end{array}$ & 0.57 & .456 \\
\hline English word attack & & & $\begin{array}{l}38.24 \\
(4.77)\end{array}$ & $\begin{array}{l}38.36 \\
(3.16)\end{array}$ & 0.10 & .919 \\
\hline Sight word efficiency & & & $\begin{array}{l}98.92 \\
(3.93)\end{array}$ & $\begin{array}{l}99.45 \\
(6.74)\end{array}$ & 0.10 & .758 \\
\hline Phonemic decoding efficiency & & & $\begin{array}{l}55.42 \\
(5.96)\end{array}$ & $\begin{array}{l}56.80 \\
(4.40)\end{array}$ & 0.74 & .396 \\
\hline
\end{tabular}

Note: Numbers inside the parentheses represent standard deviations. The scores are the number of correct items. The Chinese word efficiency (104 items) and identification tasks (40 items) were designed by authors of this study. The visual-auditory learning (134 items), English word identification (106 items), and English word attack (45 items) were subtests of Woodcock Reading Mastery Tests - Revised (WRMT-R); the sight word efficiency (104 items) and phonemic decoding efficiency (63 items) were subtests of Test of Word Reading Efficiency (TOWRE); The 
nonword repetition (18 items) was a subtest of Comprehensive Test of Phonological Processing (CTOPP). 
Table 2 Brain regions for addressed and assembled phonologies in native Chinese and native English speakers

\begin{tabular}{|c|c|c|c|c|c|c|c|c|c|c|}
\hline \multirow{2}{*}{ Brain regions } & \multicolumn{5}{|c|}{ Native Chinese speakers } & \multicolumn{5}{|c|}{ Native English speakers } \\
\hline & $\mathrm{x}$ & $\mathrm{y}$ & $\mathrm{z}$ & $\mathrm{Z}$ & Cluster size & $\mathrm{x}$ & $\mathrm{y}$ & $\mathrm{Z}$ & $\mathrm{Z}$ & Cluster size \\
\hline \multicolumn{11}{|c|}{ Addressed phonology $>$ assembled phonology } \\
\hline Left OFC/IFG & -48 & 18 & -16 & 4.39 & 3091 & & & & & \\
\hline Right OFC/IFG & 40 & 22 & -4 & 4.03 & 937 & 36 & 18 & -10 & 4.07 & 568 \\
\hline $\mathrm{ACC}$ & -8 & 34 & 28 & 4.42 & 5214 & -6 & 34 & 12 & 4.04 & 2226 \\
\hline $\mathrm{PCC}$ & -2 & -40 & 40 & 4.58 & 2276 & 6 & -40 & 22 & 3.63 & 760 \\
\hline Left MTG/AG & -66 & -22 & -8 & 4.04 & 2971 & & & & & \\
\hline Right MTG/AG & 66 & -38 & -16 & 3.85 & 1865 & 50 & -40 & 8 & 3.40 & 1230 \\
\hline Right AG & 42 & -60 & 54 & 3.62 & 1021 & & & & & \\
\hline Right FG & & & & & & 30 & -72 & -16 & 3.54 & 658 \\
\hline \multicolumn{11}{|c|}{ Assembled phonology $>$ addressed phonology } \\
\hline Left SMG/SPL & & & & & & -38 & -38 & 40 & 3.07 & 544 \\
\hline
\end{tabular}

Note: Cluster size is the number of voxels. OFC $=$ orbital frontal cortex; IFG = inferior frontal gyrus; $\mathrm{ACC}=$ anterior cingulate cortex $\mathrm{PCC}=$ posterior cingulate cortex; $\mathrm{MTG}=$ middle temporal gyrus; $\mathrm{AG}=$ angular gyrus; $\mathrm{FG}=$ fusiform gyrus; $\mathrm{SMG}=$ supramarginal gyrus; and $\mathrm{SPL}$ $=$ superior parietal lobule. 


\section{Figure captions}

Fig.1. Experimental design and examples of the stimuli. The artificial language was created by adopting the visual forms and sounds of 60 Korean Hangul characters. For both native Chinese and native English speakers, two matched groups of subjects received addressed- and assembled-phonology training for eight days (one hour per day) (A). After training, subjects were scanned when performing a naming task, in which subjects were asked to read each word aloud (B) or not to respond if they did not know the word.

Fig.2. Behavioral performance. The upper panel shows accuracies of trained words in the addressed group (A), trained words in the assembled group (B), and untrained words in the assembled group (C) during eight days of training. The lower panel shows reaction times (A) and accuracies (B) of trained and untrained artificial words during scanning. Error bars represent the standard error of the mean. TW_ADDR = trained words in the addressed group; TW_ASSE = trained words in the assembled group; UTW_ASSE = untrained words in the assembled group; and D = day.

Fig.3. Neural pathways of addressed and assembled phonologies in native English (A) and native Chinese speakers (B). Red indicates regions showing more activation for addressed phonology than assembled phonology, whereas blue indicates the reverse (assembled $>$ addressed phonology). Yellow in Panel $\mathrm{C}$ indicates the common regions for addressed phonology in native Chinese and native English speakers. All activations were thresholded at $\mathrm{z}>2.3$ (whole-brain corrected) and rendered onto PALS-B12 atlas (Van Essen, 2002, 2005) via average fiducial mapping using caret 
software (Van Essen et al., 2001). R = right.

Fig.4. Two brain regions showing significant native-language-by-training interaction. The left supramarginal gyrus showed greater differences of assembled minus addressed phonology for native English speakers than native Chinese speakers, whereas the left middle temporal gyrus showed greater differences of addressed minus assembled phonology for native Chinese speakers than native English speakers. Activations in the left and right inferior occipital gyrus were examined for scanner effects and none was found. All activations were thresholded at $\mathrm{z}>2.3$ (whole-brain corrected) and rendered onto PALS-B12 atlas (Van Essen, 2002, 2005) via average fiducial mapping using caret software (Van Essen et al., 2001). Bar graphs in the upper panel were percent signal changes in four regions, i.e., the left supramarginal gyrus, middle temporal gyrus, and bilateral inferior occipital gyrus. Error bars represent the standard error of the mean. $\mathrm{CHI}=$ native Chinese speakers; $\mathrm{ENG}=$ native English speakers; and $\mathrm{R}=$ right. 
A

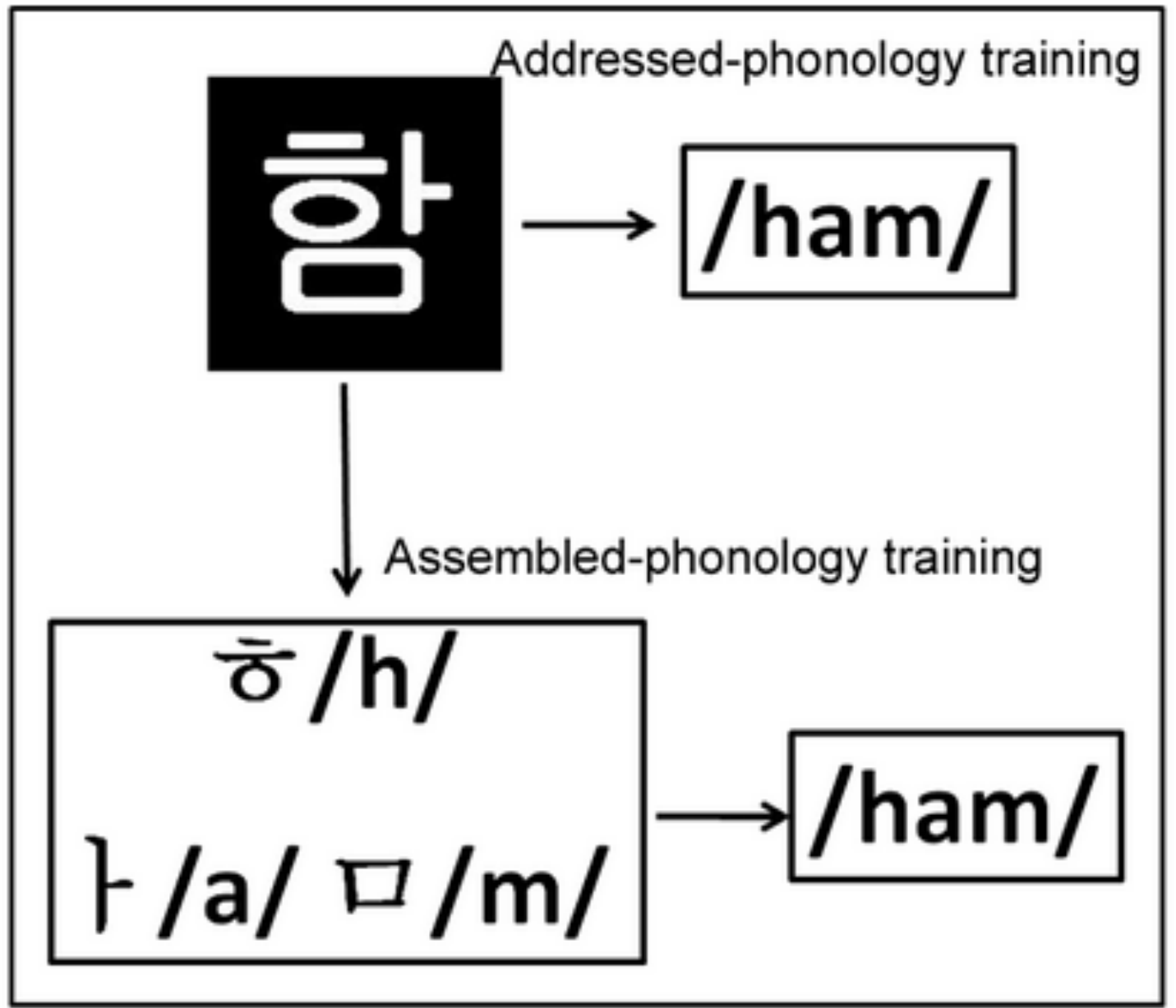

B

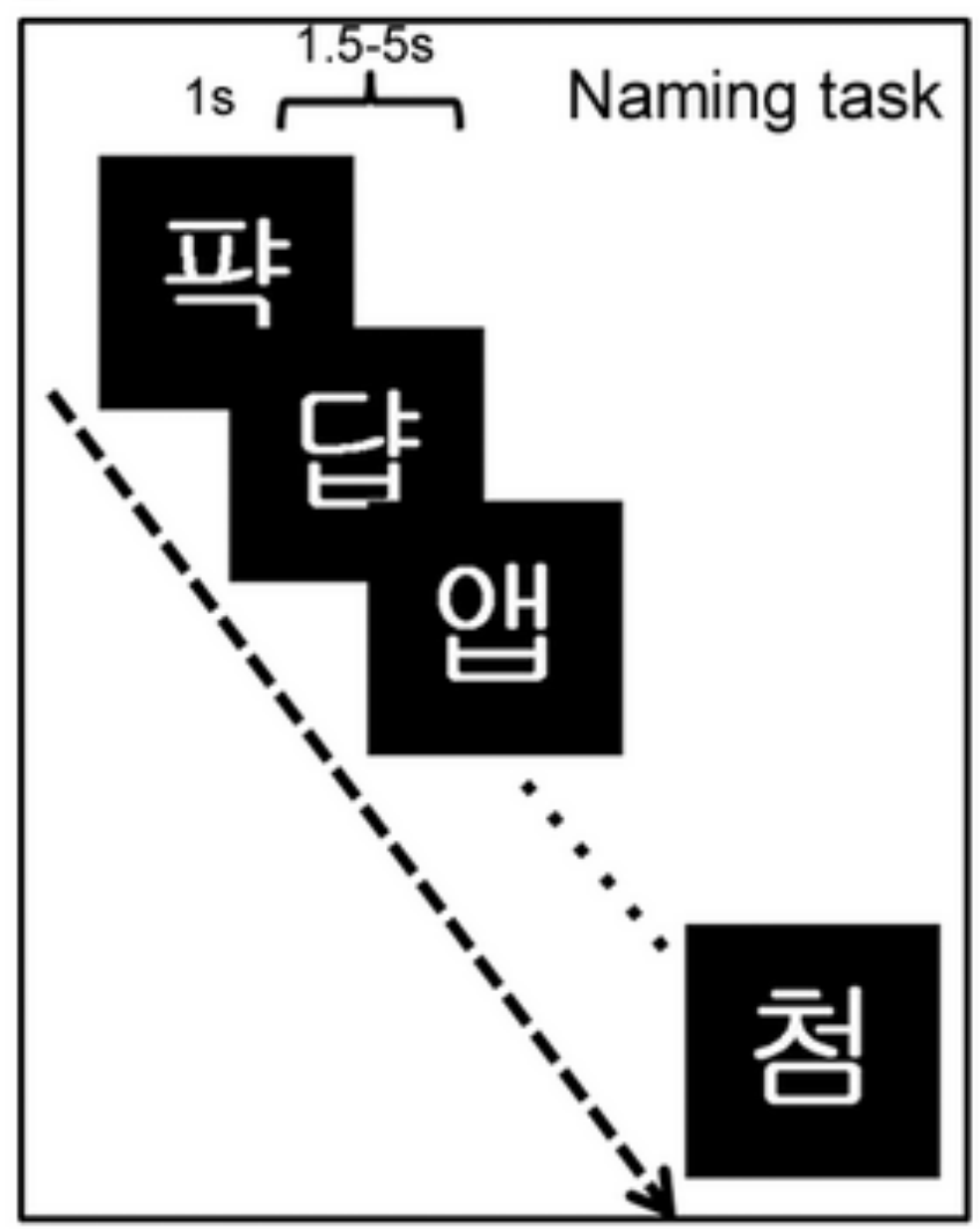




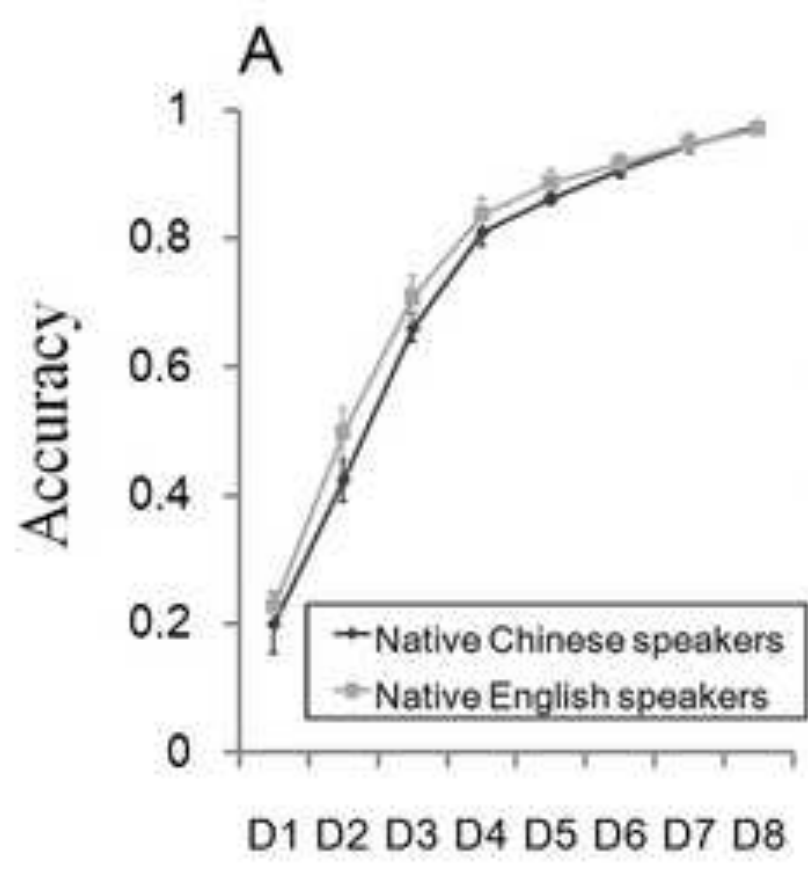

B

C
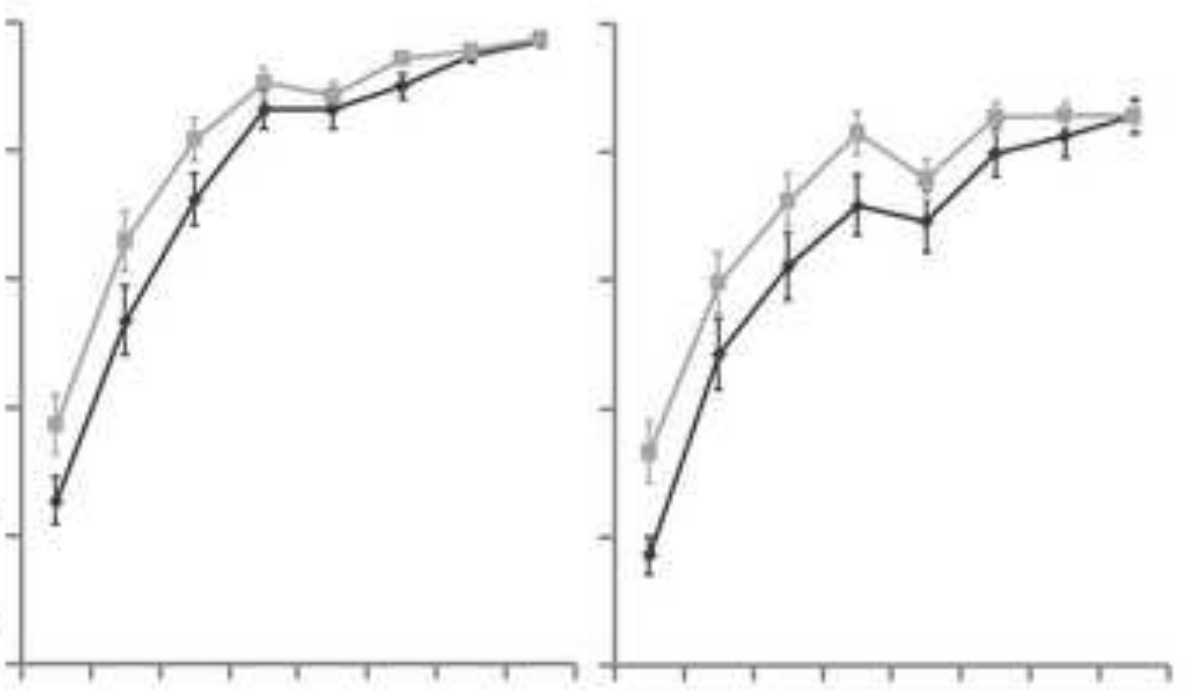

D1 D2 D3 D4 D5 D6 D7 D8

D1 D2 D3 D4 D5 D6 D7 D8

D
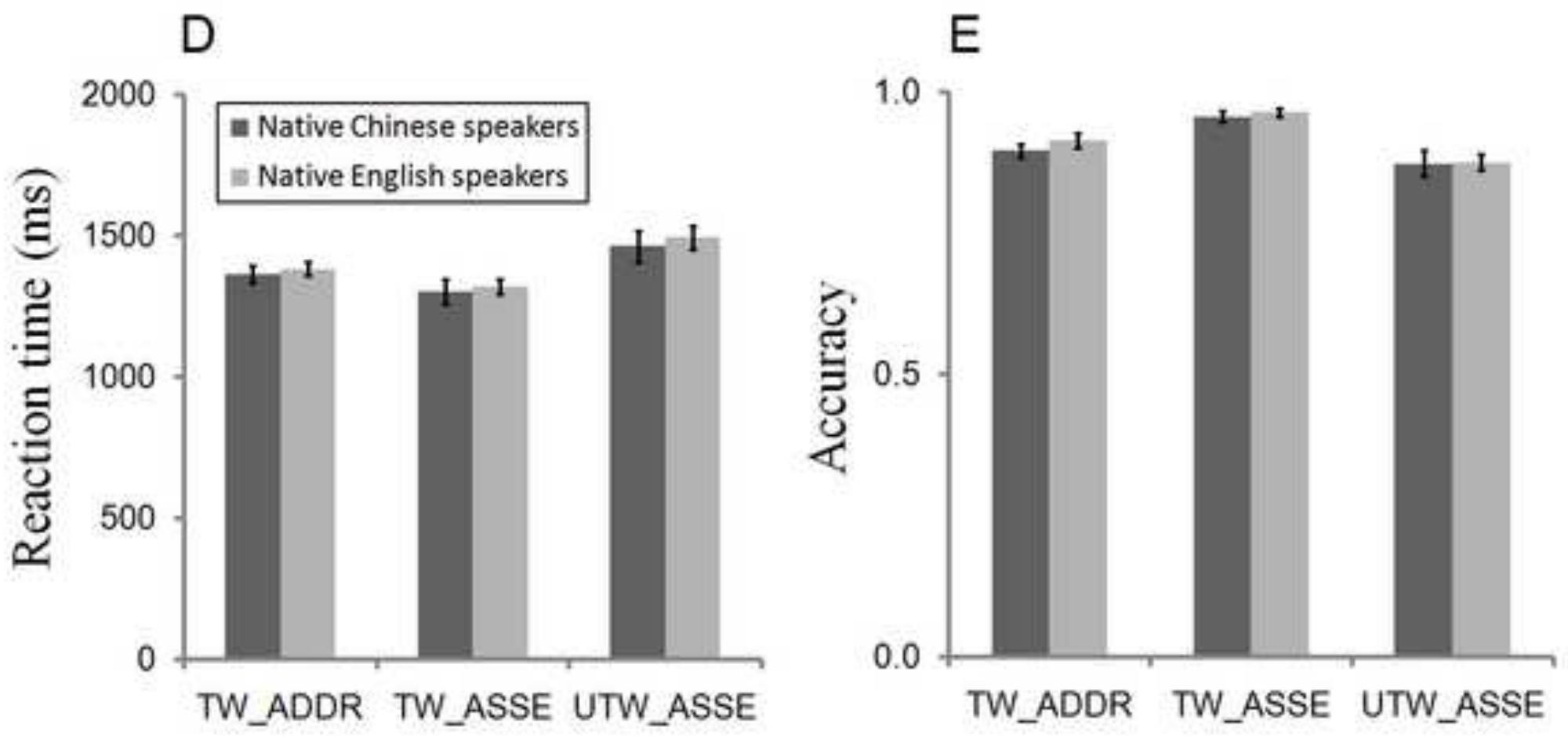
A Native English speakers
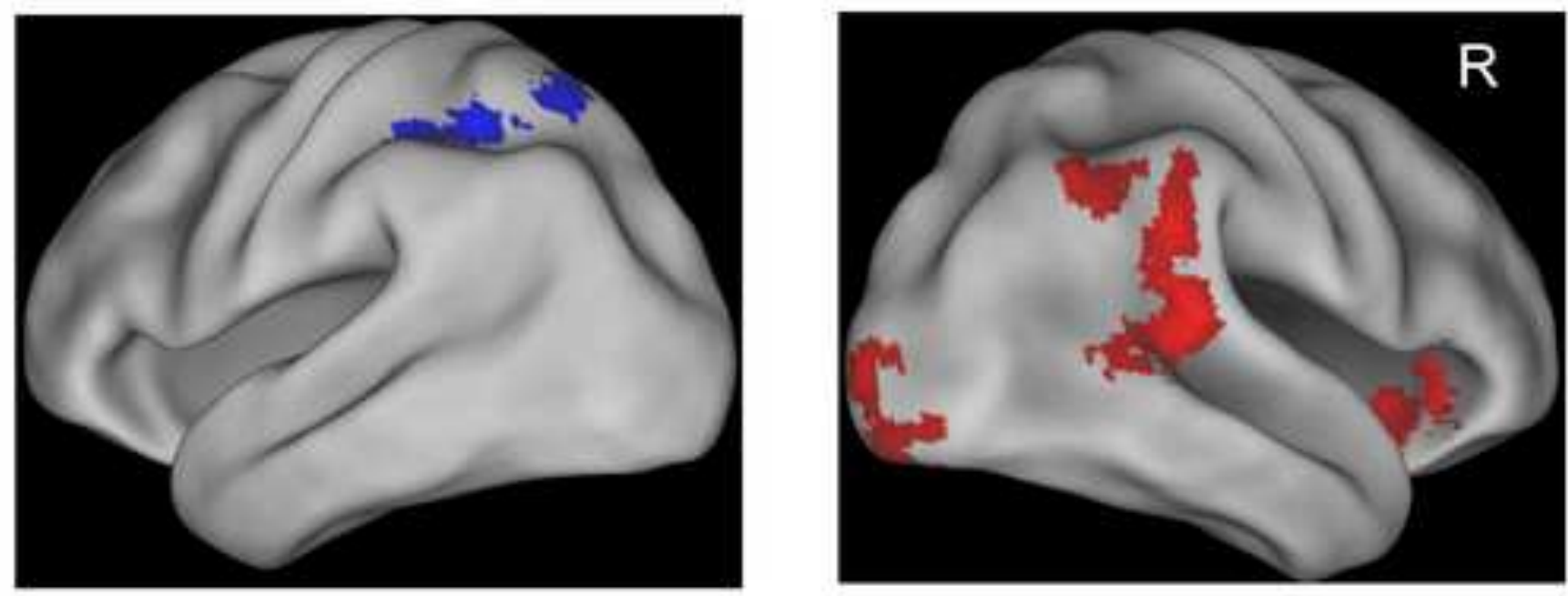

B Native Chinese speakers
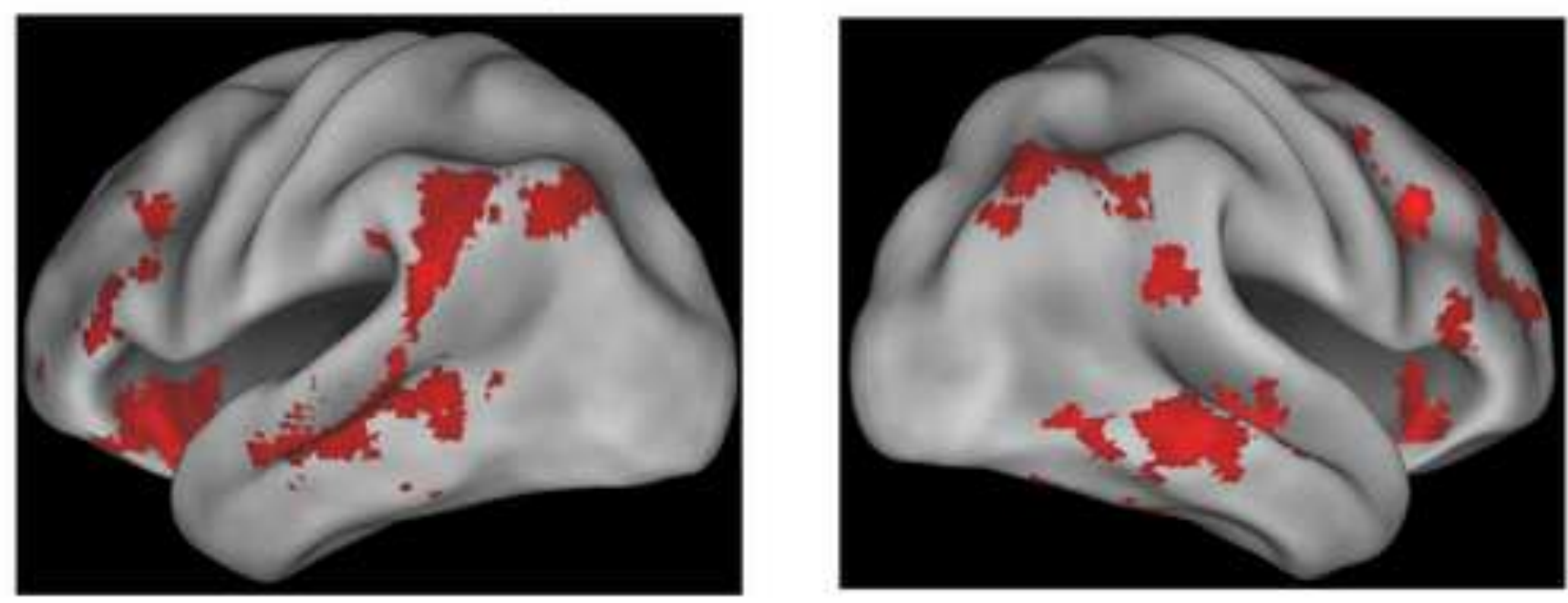

C Conjunction analysis
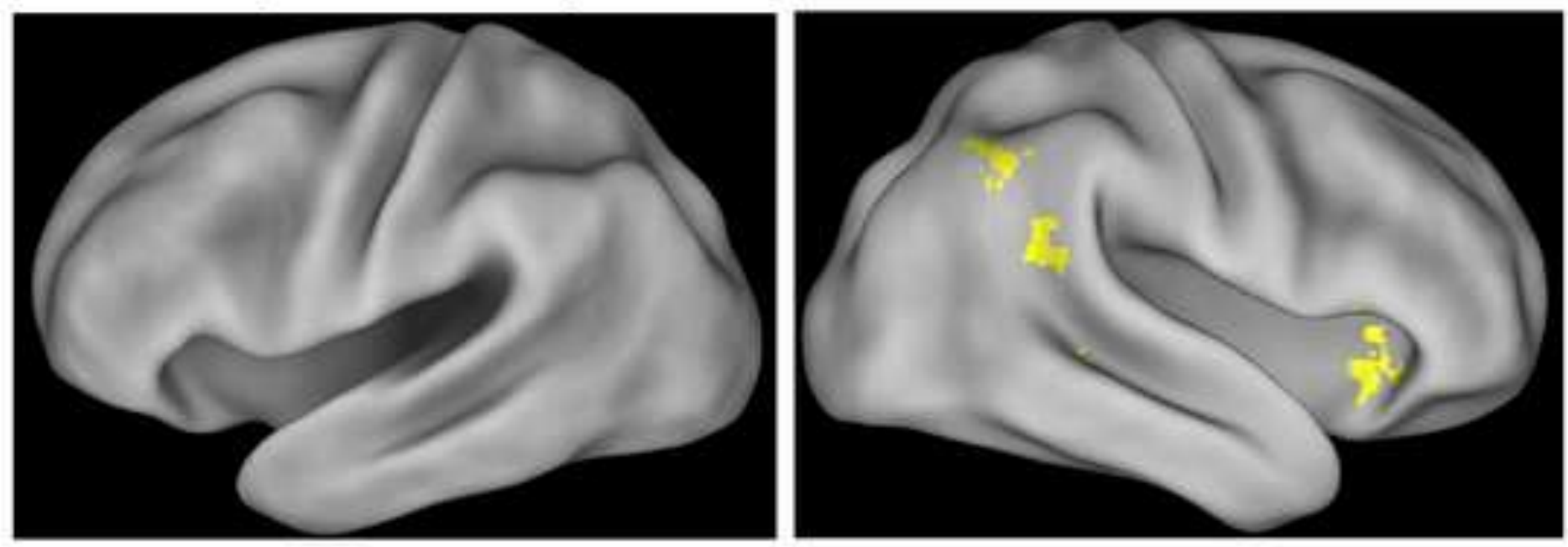

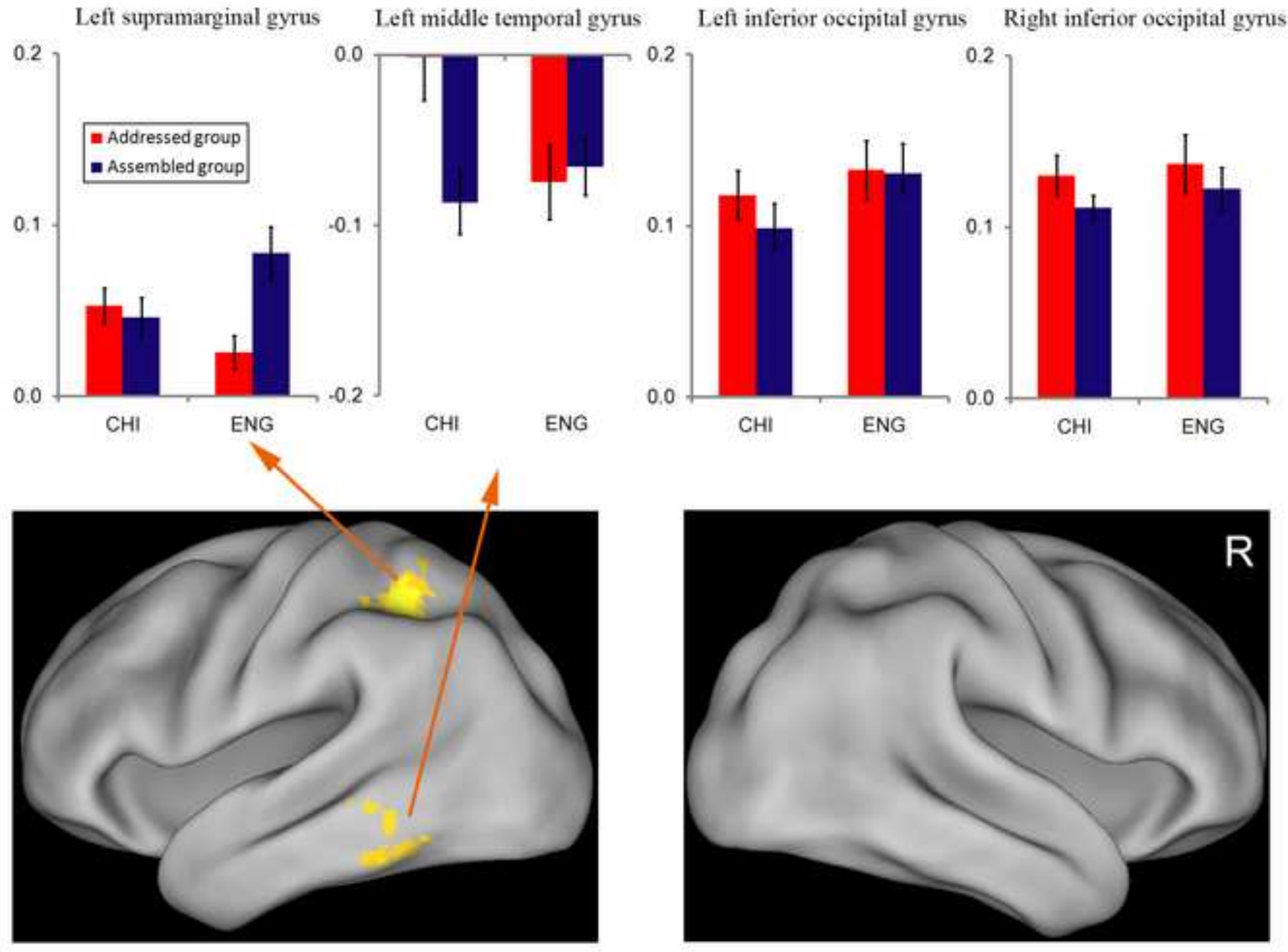